\title{
Molecular studies of non-disjunction in trisomy 16
}

\author{
T J Hassold, D Pettay, S B Freeman, M Grantham, N Takaesu
}

\begin{abstract}
The origin of the additional chromosome in 26 trisomy 16 spontaneous abortions was studied using DNA probes for chromosome 16, including a probe for centromeric $\alpha$ satellite sequences. We were able to determine the parent and meiotic stage of origin of trisomy in 22 cases, with all being attributable to maternal meiosis I non-disjunction. Furthermore, in each of the remaining four cases the results were compatible with this origin. Thus, it is likely that the high incidence of trisomy 16 results from an abnormal process acting at maternal meiosis I which more frequently involves chromosome 16 than other similar sized chromosomes. In studies of recombination, we found little evidence for an association between reduced or absent recombination and chromosome 16 non-disjunction; however, we were unable to rule out an effect of hyperrecombination.
\end{abstract}

Trisomy 16 is the most commonly identified trisomy in man, occurring in at least $1 \%$ of all clinically recognised pregnancies. ${ }^{1}$ Despite its high frequency, little is known about the parent or meiotic stage of origin of the additional chromosome. Results of chromosome heteromorphism analyses have implicated maternal non-disjunction as the most likely source of trisomy, ${ }^{1}$ but these studies have been hindered by the imprecision of the cytogenetic technique and the low level of polymorphism associated with the chromosome 16 cytogenetic marker.

We recently initiated DNA marker studies to determine the parent and meiotic origin of the extra chromosome in trisomy 16 spontaneous abortions and to assess the possibility that hypo- or hyper-recombination is associated with the non-disjunctional event. In the present report, we summarise our observations on an initial series of 26 fetuses and show that virtually all trisomy 16 results from nondisjunction at maternal meiosis I.

Division of Medical Genetics, Department of Pediatrics, Emory University School of Medicine, 2040 Ridgewood Drive, Atlanta, Georgia 30322, USA.

T J Hassold, D Pettay, S B Freeman, M Grantham, N Takaesu

Correspondence to Dr Hassold.

Received for publication 27 July 1990

Revised version accepted for publication 10 September 1990.

\section{Materials and methods}

The study population consisted of 26 trisomy 16 abortuses, 12 of which were ascertained in a cytogenetic survey of spontaneous abortions conducted in Hawaii and 14 in a similar study now under way at Emory University, Atlanta, GA. Preliminary results on the 12 Hawaiian cases were reported previously ${ }^{2}$; an additional three cases described in that report were excluded from the present study because of insufficient proband DNA. The methodology for the collection, culturing, and cytogenetic analysis of tissue samples is almost identical for the Hawaii and Atlanta studies, and has been described in detail elsewhere. ${ }^{3}$

DNA was extracted from fetal tissue and parental blood samples and processed for DNA hybridisation studies using previously described techniques. ${ }^{4}$ The parental origin of the additional chromosome was evaluated with eight probes detecting restriction fragment length polymorphisms at chromosome 16 loci: pEKMAD2.1 (D16S83), CMM65 (D16S84), 16/32 (D16S35), 16/12 (D16S36), hp2alpha (HP), pEKXp3B (CTRB), p79-2-23 (D16S7), and pHUAPRT (APRT).

The meiotic stage of origin of trisomy was studied with pSE16-2 (D16Z2), which detects highly polymorphic $\alpha$ satellite sequences at the centromere of chromosome $16^{6}{ }^{6}$ For this analysis, DNA samples from the trisomic fetus and parents were digested with $E c o R V$ and processed for hybridisation studies as for the other probes. Non-disjunction was scored as being of meiosis I origin if all centromeric restriction fragments in the parent of origin were present in the trisomic fetus, and of meiosis II origin if only a subset of the fragments were present in the fetus. This scoring system assumes that the parent of origin is heterozygous; however, this seems a safe assumption, since (1) Greig et at $t^{6}$ were unable to detect identical pSE16-2/EcoRV restriction patterns among approximately 30 unrelated persons, and (2) in the present study, in 22 of 23 cases in which the parental origin of trisomy was determined, the trisomic fetus clearly inherited only a subset of the centromeric restriction fragments from the parent who had contributed a single chromosome 16.

For trisomies in which we were able to determine the parent and meiotic stage of origin, we evaluated the frequency of crossing over between the two nondisjoined chromosomes by (1) identifying heterozygous loci in the parent of origin of trisomy, (2) 
studying these loci in the trisomic offspring to determine if heterozygosity had been maintained, or had been reduced to homozygosity, and (3) comparing the different loci in the trisomic offspring for evidence of recombination. For example, if the parent of origin were heterozygous at two loci and the trisomic offspring heterozygous at one and homozygous at the other, a single (or odd number of) crossover(s) must have occurred between these loci.

\section{Results}

The results of the DNA marker studies are summarised in table 1 and examples of determinations of parental and meiotic stage of origin of trisomy are shown in figs 1 and 2. We were able to determine both the parent and meiotic stage of origin of non-disjunction in 21 cases of simple trisomy 16 and in one case (S9) with a $47, \mathrm{XX},+16 / 48, \mathrm{XX},+2,+16$ chromosome constitution. In all 22 cases, the trisomy arose from non-disjunction at maternal meiosis $I$.

We were also able to obtain some information on each of the four remaining trisomies. In K3021, a $45, \mathrm{X} / 46, \mathrm{X},+16$ mosaic, the additional chromosome was maternally derived and, as maternal heterozygosity at two loci was maintained in the trisomic fetus, the trisomic line must have originated in meiosis. However, the centromeric marker was uninformative and therefore we were not able to distinguish between a meiosis I and II error. In S9, we were unable to determine the parental origin of trisomy, but all parental restriction fragments detected by the centromeric probe were present in the fetus. Thus, the additional chromosome 16 presumably resulted from non-disjunction at meiosis I. In S374 and S382, parental origin studies were uninformative. However, in each case the fetus had inherited all of the maternal, but only a subset of the paternal, centromeric restriction fragments. Thus, for these two cases the trisomy must have originated from an error at maternal meiosis I or at paternal meiosis II.

Table 1 also summarises the results of our analyses of recombination between the non-disjoined chromosomes. We detected recombination in 17 of the 22 cases involving maternal meiosis I non-disjunction; in six of these we identified two crossovers and in the other 11 cases one crossover. We were unable to detect recombination in the other five cases involving

Table 1 Summary of DNA marker studies of 26 spontaneous abortions with trisomy 16.

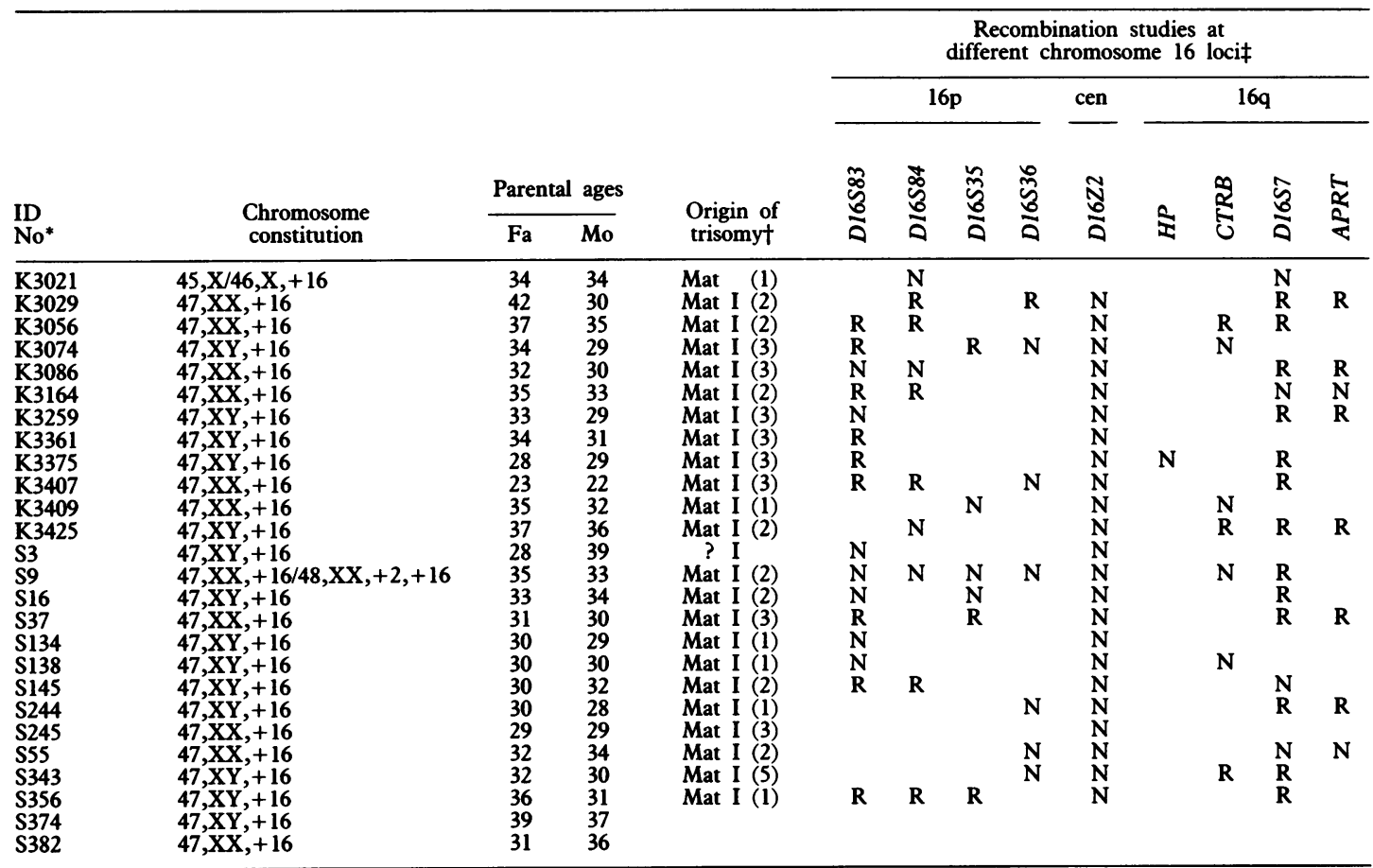

*Samples with a $\mathrm{K}$ prefix were ascertained in a cytogenetic study of spontaneous abortions in Honolulu; those with an $\mathrm{S}$ prefix were ascertained in a similar study in Atlanta.

tNo in ()$=$ number of markers informative for parental origin of trisomy.

$\ddagger$ For loci heterozygous in parent of origin, trisomic fetus is scored as $\mathbf{N}$ (non-reduced) if heterozygosity is maintained, or $\mathbf{R}$ (reduced) if heterozygosity is converted to homozygosity. Crossing over must have occurred between adjacent loci whenever one is $\mathbf{N}$ and the other $\mathbf{R}$. 


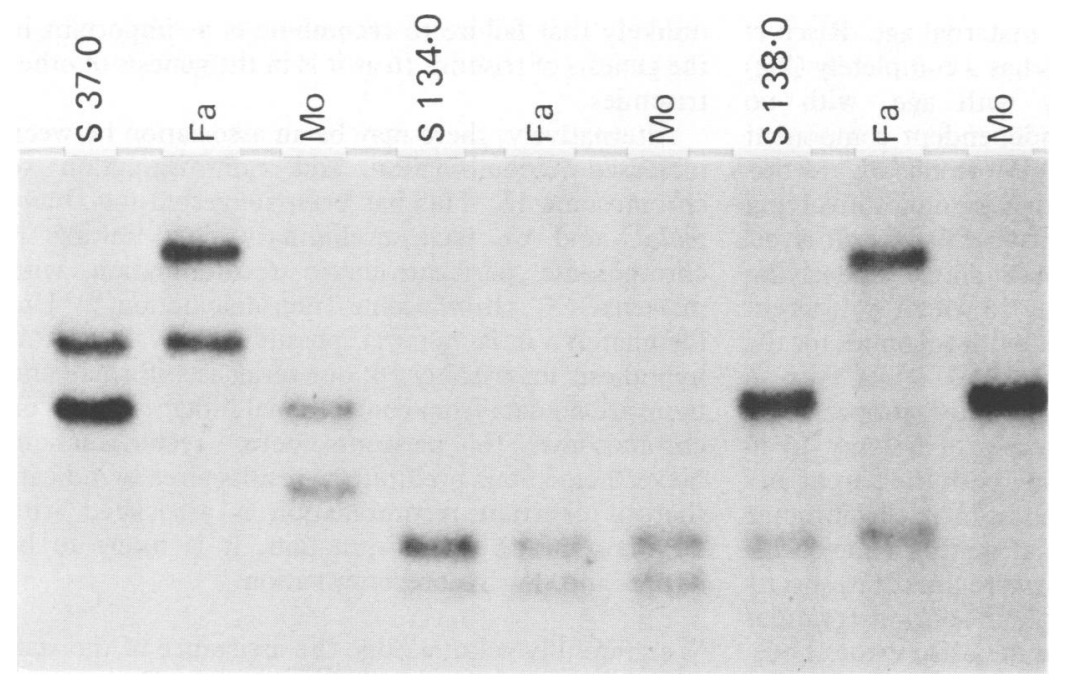

Figure 1 DNA marker studies of parental origin of trisomy 16. DNA from three trisomy 16 spontaneous abortions ( $S 37.0$, S134.0, S138.0) and their parents was digested with TaqI and probed with p79-2-23, which detects multiallelic variation at D16S7.7 Results are consistent with maternal nondisjunction for $S 37.0$ and $S 138.0$ since both have inherited a single allele from the father and, by dosage, two copies of a maternal allele. An effect of dosage is evident also for S134.0; however, as the parents are heterozygous for the same alleles, a parental origin determination cannot be made.

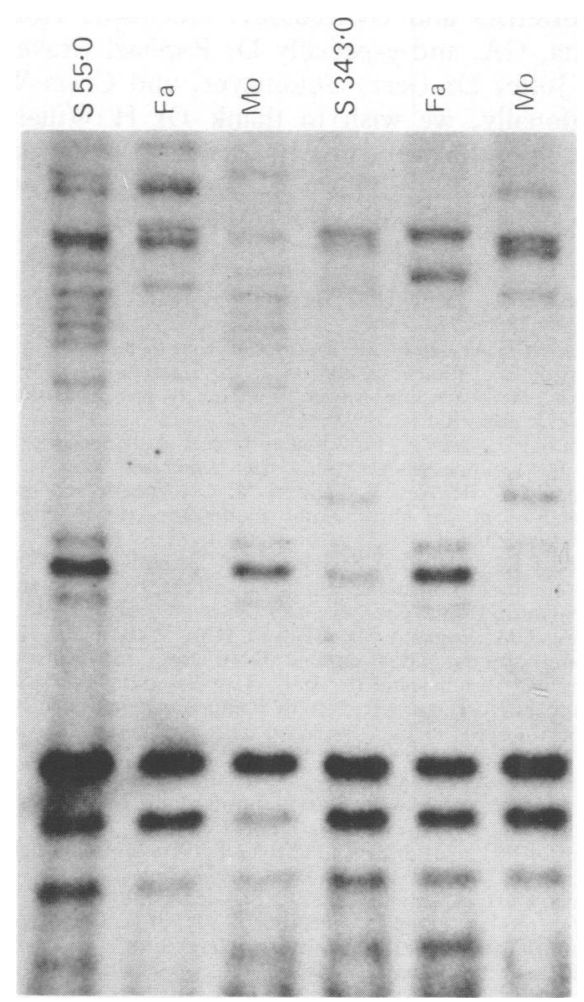

Figure 2 Analysis of the meiotic stage of chromosome 16 nondisjunction. DNA from two trisomy 16 spontaneous abortions (S55.0 and S343.0), known from other markers to be maternally derived trisomies, and their parents were digested with EcoRV and probed with pSE 16-2, which detects a satellite variation at the centromere of chromosome $16 .^{6}$ Each fetus has inherited a subset of the father's restriction fragments but all of the mother's restriction fragments. Assuming the mother to be heterozygous, the results of the centromere marker studies indicate that non-disjunction occurred at maternal meiosis $I$.
Table 2 Evidence for recombination in maternally derived sex chromosome trisomies, trisomy 16, and trisomy 21 .

\begin{tabular}{lcc}
\hline & \multicolumn{2}{c}{ Evidence for recombination* } \\
\cline { 2 - 3 } Trisomy & Yes & No \\
\hline XXY, XXX & 22 & $14(39 \%)$ \\
16 & 9 & $0(0 \%)$ \\
21 & 16 & $12(43 \%)$ \\
\hline
\end{tabular}

*In this analysis, we considered only those trisomies which were informative for recombination at four or more non-centromeric loci. Trisomies were scored as positive for recombination if at least one crossover was detected and negative if informative loci were nonreduced. Data on XXYs and XXXs are from Morton $e t a l^{12}$ and unpublished observations; data on trisomy 21 are from unpublished observations.

maternal meiosis I errors, but in most of these there were only one or two informative loci.

\section{Discussion}

Trisomy 16 is unusual in at least two respects: its incidence and its relationship to maternal age. It is extraordinarily common, occurring in 7 to $8 \%$ of all spontaneous abortions and, by extrapolation, over $1 \%$ of all clinically recognised human pregnancies. ${ }^{1}$ The reason for this high frequency is unknown, but it is more likely to result from variation in non-disjunctional frequency than differential in utero selection among trisomies. That is, trisomy 16 is identified more frequently than are trisomies $13,18,21$, or the sex chromosome trisomies, despite the fact that the latter trisomies are compatible with livebirth. Furthermore, even among spontaneous abortions, trisomy 16 is not characteristically associated with late fetal wastage. Thus, it seems likely that, at the time of conception, trisomy 16 is disproportionately represented. This implies that there are mechanisms of non-disjunction largely, or wholly, restricted to chromosome 16 , and 
this is consistent with data on maternal age. Risch $e t$ $a l^{8}$ have shown that trisomy 16 has a completely (log) linear increase in frequency with age, with no evidence for a maternal age independent component and this has been confirmed by Morton et al. ${ }^{9}$ In this respect, trisomy 16 is unlike other trisomies involving similar sized chromosomes. From this, Risch $e t a l^{8}$ suggested that there may be a single underlying mechanism of origin for trisomy 16 which is different from the mechanisms associated with trisomies for the other small chromosomes.

The results of the present study support this interpretation. In each of 22 cases of trisomy 16 in which we were able to determine both the parent and meiotic stage of origin, the additional chromosome resulted from non-disjunction at maternal meiosis I. In four other cases in which we were unable to specify the parent or stage of origin or both, our observations were consistent with a maternal meiosis I error. Thus, in all 26 cases our results were compatible with maternal meiosis I non-disjunction. Maternal errors predominate in other trisomies that have been studied with DNA markers, ${ }^{2}{ }^{10}{ }^{11}$ but to our knowledge no other trisomy has as high a proportion of cases of maternal origin. Thus, it may be that the high incidence of trisomy 16 is attributable to an abnormal process acting at maternal meiosis I which is more likely to disrupt disjunction of chromosome 16 than other chromosomes.

In the second aspect of our study, we were interested in determining whether or not chromosome 16 non-disjunction might be related to abnormally low or high levels of recombination. Recent studies from our laboratory and others suggested an association between failure to recombine and maternal meiosis I non-disjunction for the $\mathrm{XX}^{12}$ and chromosome 21 bivalents $^{13}$ (Hassold, unpublished observations). Therefore, we examined our cases for possible pairing/recombination failure, but found little evidence for such an effect. We identified recombinants in each of the nine cases having four or more informative, non-centromeric loci; overall, we identified recombinants in 17 of the 22 cases attributable to maternal meiosis I non-disjunction. This contrasts markedly with our observations for maternally derived sex chromosome trisomies and trisomy 21 , in which a significant proportion of cases have no detectable recombination between the non-disjoined chromosomes (table 2). It may be possible to explain the difference between trisomies 16 and 21 by invoking the different genetic lengths of the two chromosomes; that is, since the chromosome 21 map is smaller we would expect a higher proportion of apparent nonrecombinants for trisomy 21 than trisomy 16 . However, this cannot explain the difference between trisomy 16 and the sex chromosome trisomies, since the female $\mathrm{X}$ chromosome map is at least as long as the map for chromosome $16 .{ }^{14}$ Therefore, it seems unlikely that failure to recombine is as important in the genesis of trisomy 16 as it is in the genesis of other trisomies.

Alternatively, there may be an association between increased recombination and non-disjunction of chromosome 16. This has been suggested for Drosophila $^{15}$ and we have preliminary data linking $X$ chromosome pericentromeric recombination with maternal $\mathrm{X}$ chromosome non-disjunction. ${ }^{12} \mathrm{Un}$ fortunately, it is not yet possible to evaluate this hypothesis for trisomy 16; our series is still small and there are no data from conventional linkage studies on chromosome 16 pericentromeric recombination. Nevertheless, our preliminary results already indicate that, if aberrant recombination is associated with chromosome 16 non-disjunction, it is likely to be hyper- and not hyporecombination.

We gratefully acknowledge the assistance of the staff of the Department of Pathology and the Department of Obstetrics and Gynecology, Northside Hospital, Atlanta, GA, and especially Dr Raphael Graves, Dr Alan Joffe, Dr Gerry Sotomayer, and Chris Wylie. Additionally, we wish to thank $\mathrm{Dr} \mathrm{H}$ Willard for donation of the probe pSE16-2, and for his comments on the manuscript. This work was supported by NIH grants HD 21341 and HD 25509.

1 Hassold T, Jacobs PA. Trisomy in man. Annu Rev Genet 1984;18:69-97.

2 Hassold T, Takaesu N. Analysis of non-disjunction in human trisomic spontaneous abortions. In: Hassold TJ, Epstein CJ, eds. Molecular and cytogenetic studies of non-disjunction. New York: Alan R Liss, 1989:115-34.

3 Hassold T, Chen N, Funkhouser J, et al. A cytogenetic study of 1,000 spontaneous abortions. Ann Huit Genet 1980;44:151-78.

4 Hassold T, Benham F, Leppert M. Cytogenetic and molecular analysis of sex chromosome monosomy. Am $\mathcal{f}$ Hum Genet 1988;42:534-41.

5 Kidd KK, Bowcock AM, Schmidtke J, et al. Report of the DNA committee and catalogs of cloned and mapped genes and DNA polymorphisms. Cytogenet Cell Genet 1989;51:622-947.

6 Greig GM, England SB, Bedford HM, Willard HF. Chromosome-specific alpha satellite DNA from the centromere of human chromosome 16. Am f Hum Genet 1989;45:862-72.

7 Chimera JA, Harris CR, Litt M. Population genetics of the highly polymorphic locus D16S7 and its use in paternity evaluation. Am F Hum Genet 1989;45:926-31.

8 Risch N, Stein Z, Kline J, Warburton D. The relationship between maternal age and chromosome size in autosomal trisomy. Am $\mathcal{F}$ Hum Genet 1986;39:68-78.

9 Morton NE, Jacobs PA, Hassold T, Wu D. Maternal age in trisomy. Ann Hum Genet 1988;52:227-35.

10 Chakravarti A, Majumder PP, Slaugenhaupt SA, et al. Genecentromere mapping and the study of non-disjunction in autosomal trisomies and ovarian teratomas. In: Hassold TJ, Epstein CJ, eds. Molecular and cytogenetic studies of nondisjunction. New York: Alan R Liss, 1989: 45-79.

11 Kupke KG, Muller U. Parental origin of the extra chromosome in trisomy 18. Am F Hum Genet 1989;45:599-605.

12 Morton NE, Keats BJ, Jacobs PA, et al. A centromere map of the $\mathrm{X}$ chromosome from trisomies of maternal origin. Ann $\mathrm{Hum}$ Genet 1990;54:39-47.

13 Warren AC, Chakravarti A, Wong C, et al. Evidence for reduced recombination on the nondisioined chromosome 21 in Down syndrome. Science 1987;237:652-4.

14 Keats B, Ott J, Conneally M. Report of the committee on linkage and gene order. Cytogenet Cell Genet 1989;51:459-502.
Merriam JR, Frost JN. Exchange and non-disjunction in female

15 Merriam JR, Frost JN. Exchange and non-disjunction 